\title{
Time-of-flight Mass Measurement of Neutron-rich Nuclei
}

\author{
Zach Meisel $^{* b d f}$, S. George ${ }^{c}$, S. Ahn ${ }^{b f}$, J. Browne ${ }^{b d f}$, D. Bazin ${ }^{d f}$, F. Carpino ${ }^{j}$, \\ H. Chung ${ }^{j}$, A. Estradé ${ }^{h}$, M. Famiano ${ }^{j}$, A. Gade ${ }^{d f}$, C. Langer ${ }^{b f}$, M. Matoš $^{g}$, W. Mittig ${ }^{d f}$, \\ F. Montes ${ }^{b f}$, D. Morrissey ${ }^{d e}$, J. Periera ${ }^{b f}$, H. Schatz ${ }^{b d f}$, J. Schatz, M. Scott ${ }^{d f}$,

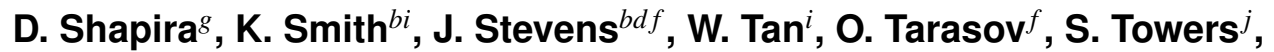 \\ K. Wimmer ${ }^{a f}$, J. Winkelbauer ${ }^{d f}$, J. Yurkon ${ }^{f}$, and R.G.T. Zegers ${ }^{b d f}$ \\ ${ }^{a}$ Department of Physics, Central Michigan University, Mt. Pleasant, MI, USA \\ ${ }^{b}$ Joint Institute for Nuclear Astrophysics, East Lansing, MI, USA \\ ${ }^{c}$ Max-Planck-Institut für Kernphysik, Heidelberg, Germany \\ ${ }^{d}$ Department of Physics and Astronomy, Michigan State University, East Lansing, MI, USA \\ ${ }^{e}$ Department of Chemistry, Michigan State University, East Lansing, MI, USA \\ ${ }^{f}$ National Superconducting Cyclotron Laboratory, East Lansing, MI, USA \\ ${ }^{g}$ Oak Ridge National Laboratory, Oak Ridge, TN, USA \\ ${ }^{h}$ School of Physics and Astronomy, The University of Edinburgh, Edinburgh, UK \\ ${ }^{i}$ Department of Physics, University of Notre Dame, South Bend, IN, USA \\ ${ }^{j}$ Department of Physics, Western Michigan University, Kalamazoo, MI, USA \\ E-mail: meisel@nscl.msu.edu
}

\begin{abstract}
Astrophysical calculations often require accurate masses of exotic nuclei. In many cases nuclear masses are critical in obtaining a reliable understanding of observational data. The time-of-flight method provides a way to measure the masses of nuclei far from the valley of beta stability with sufficient precision to address questions of stellar nucleosynthesis and nuclear structure in general. Recently such a time-of-flight experiment has been performed at the National Superconducting Cyclotron Laboratory (NSCL) where significant progress has been made on the neutron-rich side of stability in the argon to iron region. We expect to make advances in understanding nuclear structure in the region and nucleosynthesis occurring in accreted neutron star crusts.
\end{abstract}

XIII Nuclei in the Cosmos,

7-11 July, 2014

Debrecen, Hungary

\footnotetext{
* Speaker.
} 


\section{Time-of-Flight Method}

The time-of-flight method exploits our knowledge of the equation of motion of a charged particle through a magnetic system. Equating the Lorentz force with zero-electric field with the centripetal force, it is apparent that the rest mass $\left(m_{0}\right)$ of a particle with charge $q$ is proportional to the magnetic rigidity $(B \rho)$ and time-of-flight $(T O F)$ along a flight path of length $L_{p a t h}$,

$$
m_{0}=\frac{T O F}{L_{p a t h}} \frac{q(B \rho)}{\gamma} .
$$

Thus, a precise measurement of time-of-flight and magnetic rigidity yields a precise mass measurement. In practice, due to insufficient knowledge of systematic effects, the TOFs of several nuclei, including those with known mass, are measured simultaneously to result in a calibrated $T O F-m_{0}$ relationship [1, 2, 3].

\section{Experimental Set-up}

Recently a time-of-flight mass measurement (similar to the one described in [3, 4]) has been performed at the National Superconducting Cyclotron Laboratory with the use of the S800 spectrograph, as seen in Figure 1. Neutron-rich nuclei in the silicon to zinc region were produced by fragmenting a ${ }^{82} \mathrm{Se}$ beam on a beryllium target of one of two thicknesses. The thin target produced more stable nuclei required for calibration masses, where the thick target produced the more exotic nuclei of interest. Time-of-flight was measured between the extended focal plane of the A1900 fragment separator [5] and the S800 focal plane. Magnetic rigidity was measured at a dispersive focus at the entrance of the S800 spectrograph [6]. Magnetic elements in the beam-line were tuned such that the time-of-flight of a nucleus depended mostly on its mass to charge ratio $\left(m_{0} / q\right)$, with a slight momentum dependence remaining. We were able to quantify the momentum dependence of the time-of-flight with our magnetic rigidity measurement.

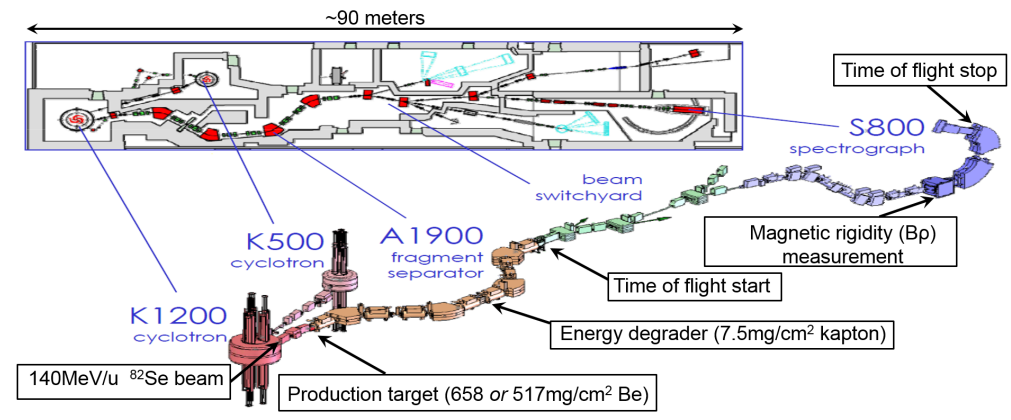

Figure 1: Experimental set-up for TOF mass measurement at the NSCL.

\section{Detectors}

BC418 fast timing scintillators from Saint Gobain Crystals [7] coupled to Hamamatsu [8] R4998 photomultiplier tubes provided timing signals for the time-of-flight measurement. Intrinsic 
timing resolution of this combination was $\sim 30$ picoseconds. The magnetic rigidity of individual ions was determined by measuring their position at the dispersive focus prior to the S800 spectrograph. For this purpose a $1500 \AA$ gold foil backed by $70 \mu \mathrm{g} / \mathrm{cm}^{2}$ mylar was placed in the path of the beam, causing electrons to be emitted from the foil by passing ions, as seen in Figure 2. The electrons were guided towards the surface of a microchannel plate detector (MCP) equipped with a resistive back-plane from Quantar Technology [9] via magnetic and electric fields. This technique had the result that the electron-impact position on the microchannel plate detector would directly correspond to the ion-impact position on the foil [10]. The microchannel plate detector amplified electrons which reached its surface, resulting in an avalanche of electrons that reached its backplane. Electrons drifted to the four corners of the back-plane, allowing for a position determination with a resolution of $\sim 0.45 \mathrm{~mm}$. The measured TOF vs dispersive MCP position was $\sim 40 \mathrm{ps} / \mathrm{mm}$, which corresponds to $\sim 120 \mathrm{~ns} / \mathrm{T}$.m. After performing the rigidity correction, the timing resolution was $\sim 75$ ps.

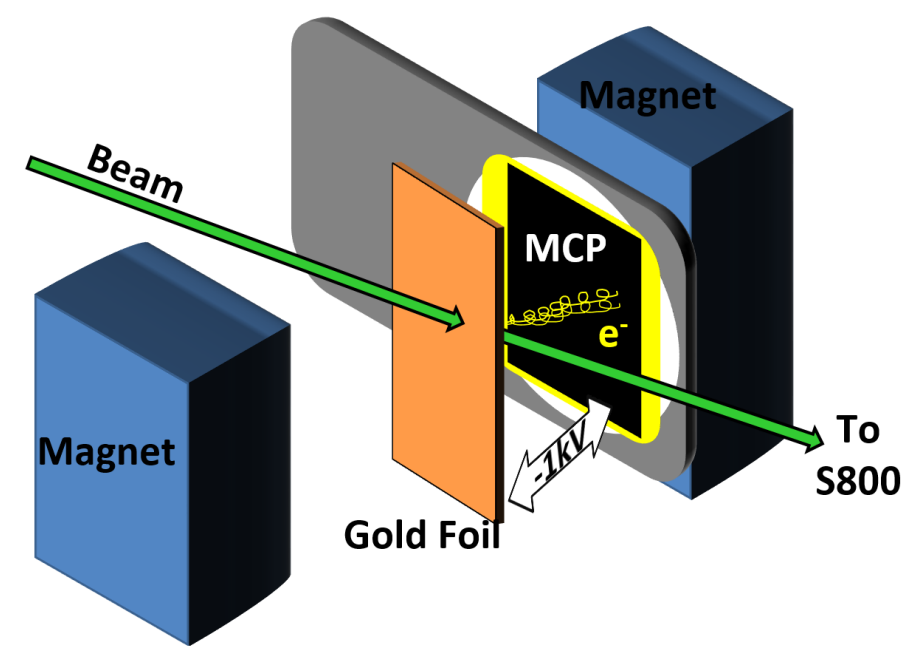

Figure 2: Position sensitive microchannel plate detection system at the dispersive focus.

\section{Analysis}

The analysis procedure consists of correcting isotopes' timing distributions for their momentumdependence, as seen in Figure 3, obtaining a relationship between momentum-corrected time-offlight and mass for nuclei of known mass, and ultimately using this relationship to obtain masses for all measured nuclei.

The time-of-flight versus microchannel plate dispersive position (TOFvsMCPx) trend was fit for each isotope of sufficient statistics. In a second step another fit was performed to arrive at a global equation for parameters of the TOF vs $M C P x$ trend, which was used for the rigidity correction of all isotopes in order to minimize the impact of systematic effects. This drastically narrowed the time-of-flight distribution of each isotope, as seen in Figure 3. 


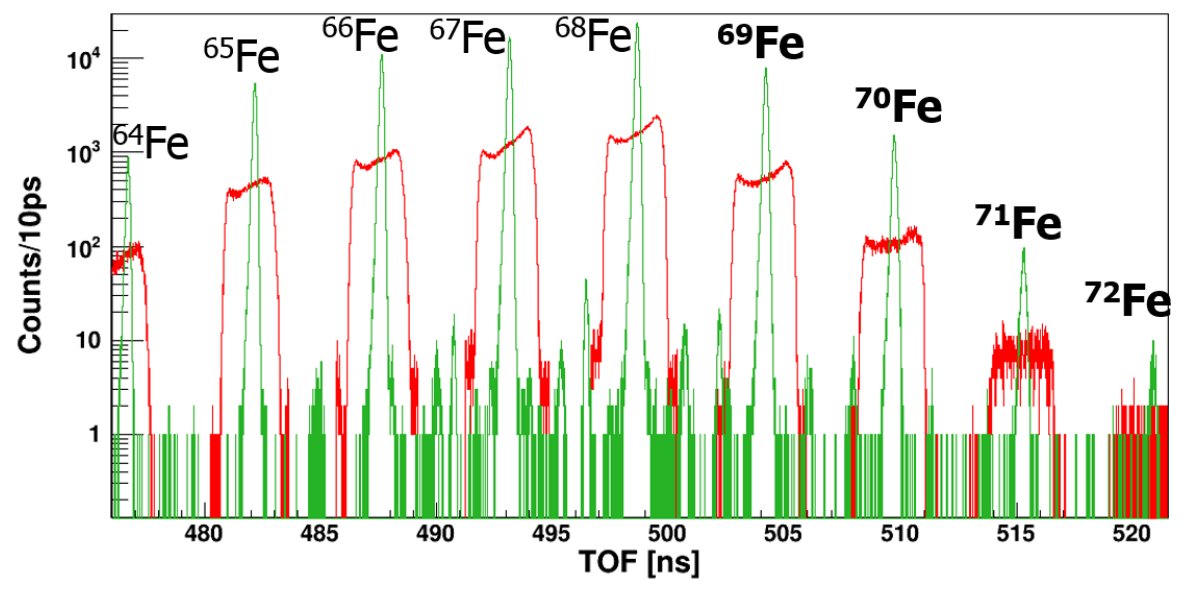

Figure 3: Time-of-flight distributions for the iron isotopes observed in this experiment before (red) and after (green) the momentum correction. Isotopes with unknown literature masses [11] have bold labels.

The relationship between mass and time-of-flight was calibrated via a fit to measured nuclei with a well-known literature mass, e.g.:

$$
m / q=f(T O F, Z)=a_{0}+a_{1} * T O F+a_{2} * T O F^{2}+a_{3} * Z+a_{4} * Z^{2}+a_{5} * T O F * Z+a_{6} * Z^{4} .
$$

A systematic uncertainty was added in to the statistical uncertainty of each nucleus involved in the mass fit until $\chi_{\text {red }}^{2} \sim 1$ was obtained;

$$
\begin{gathered}
\chi^{2}=\Sigma_{\text {calibrants }} \frac{\left((m / q)_{\text {literature }}-f(T O F, Z)\right)^{2}}{\left(\sigma_{\text {literature }}\right)_{i}^{2}+\left(\sigma_{\text {statistical }}\right)_{i}^{2}+\left(\sigma_{\text {systematic }}\right)^{2}}, \\
\text { where }\left(\sigma_{\text {statistical }}\right)_{i}^{2}=\left(\frac{\partial f(T O F, Z)}{\partial T O F}\right)^{2} * \sigma_{i}^{2}(T O F) .
\end{gathered}
$$

\section{Preliminary Results}

Several neutron-rich nuclei with unknown masses have been observed in our time-of-flight experiment, as seen in Figure 4. The time-of-flight mass measurement analysis is currently in its final stages. A precise mass-fit has been obtained for nuclei in the argon-iron range, where reference mass nuclei are reproduced within $\sim 400 \mathrm{keV}$ with the absence of systematic trends, as seen in Figure 5. In addition, we found no systematic dependencies on the production target thickness, rigidity correction method, PID-cut, timing signals, and mass-fit function chosen. Based on these results we conclude that our mass fit can be reliably extrapolated to unknown masses.

\section{Acknowledgments}

This project is funded by the NSF through grants PHY1068217 and PHY1102511. S.G. acknowledges support from the DFG under Contract No GE2183/1-1 and GE2183/2-1. 


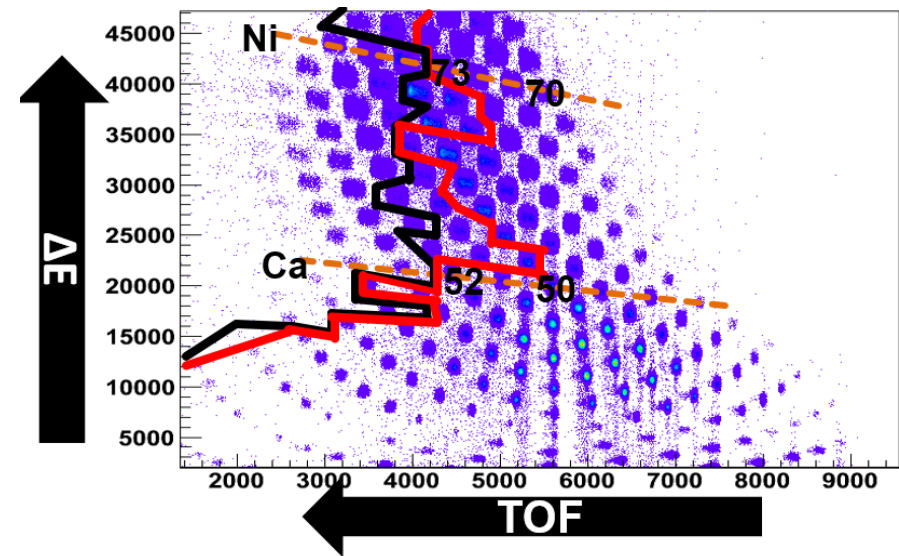

Figure 4: Particle identification (PID) plot for the recent time-of-flight mass measurement performed at the NSCL. Nuclei left of the black line have an unknown experimental mass as of the 2012 Atomic Mass Evaluation [11]. Nuclei right of the red line have a mass experimentally known to better than $200 \mathrm{keV}$ as of the 2012 Atomic Mass Evaluation.

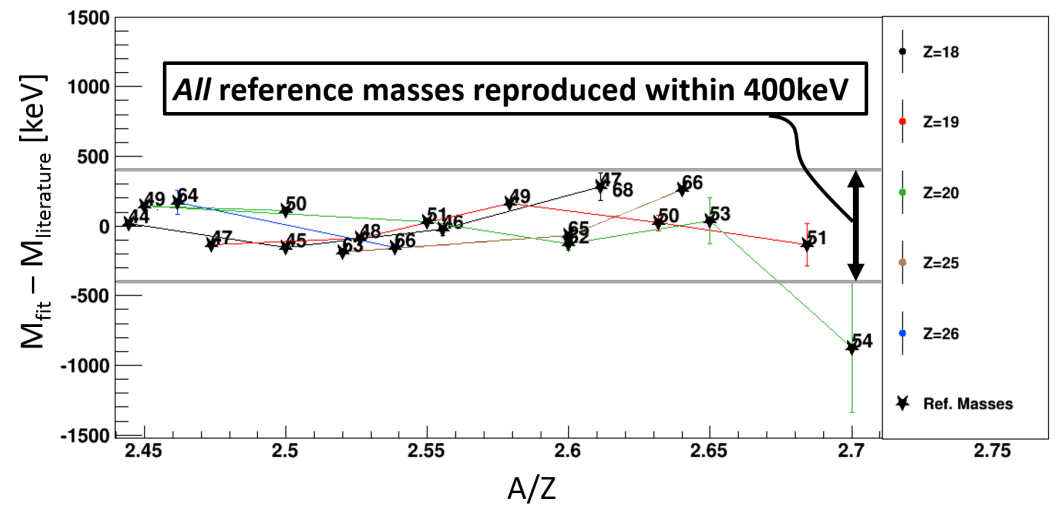

Figure 5: Fit residuals between reference nuclei and the $m_{0}(T O F)$ relationship. Reference nuclei, nuclei with literature mass uncertainties less than $100 \mathrm{keV} \mathrm{[11]} \mathrm{and} \mathrm{no} \mathrm{known} \mathrm{isomers} \mathrm{with} \mathrm{half-lives} \mathrm{longer} \mathrm{than}$ $100 \mathrm{~ns}$ [12], were all fit within $400 \mathrm{keV}$ (within their $1 \sigma$ uncertainties) with an absence of any systematic trends.

\section{References}

[1] Z. Meisel and S. George, Time-of-flight mass spectrometry of very exotic systems, IJMS 349-350, 145 (2013).

[2] M. Matoš et al. Time-of-flight mass measurements of exotic nuclei, NIMA 696, 171 (2012).

[3] A. Estradé Time-of-flight Mass Measurements of Neutron Rich Isotopes at the NSCL, Michigan State University PhD Thesis, (2010).

[4] A. Estradé et al. Time-of-Flight Mass Measurements for Nuclear Processes in Neutron Star Crusts, PRL 107, 172503 (2011).

[5] D.J Morrissey et al. Commissioning the A1900 projectile fragment separator, NIMB 204, 90 (2003). 
[6] D. Bazin et al. The S800 spectrograph, NIMB 204, 629 (2003).

[7] http://www.crystals.saint-gobain.com/, Accessed 10 September 2014.

[8] http://www.hamamatsu.com/, Accessed 10 September 2014.

[9] http://www.quantar.com/, Accessed 10 September 2014.

[10] D. Shapira, T.A. Lewis, \& L.D. Hullett, A fast and accurate position-sensitive timing detector based on secondary electron emission, Nucl.Inst.Meth. A 454, 409 (2000).

[11] G. Audi et al. The Ame2012 atomic mass evaluation, Ch.Phys.C 36, 12, 002 (2012).

[12] G. Audi et al. The Nubase2012 evaluation of nuclear properties, Ch.Phys.C 36, 12, 1157 (2012). 